\title{
Improvement of in vivo efficacy of recombinant human erythropoietin by encapsulation in PEG-PLA micelle
}

This article was published in the following Dove Press journal:

International Journal of Nanomedicine

21 December 2012

Number of times this article has been viewed

\author{
Yanan Shi ${ }^{1,2, *}$ \\ Wan Huang ${ }^{1, *}$ \\ Rongcai Liang ${ }^{1-3}$ \\ Kaoxiang Sun ${ }^{2,3}$ \\ Fangxi Zhang ${ }^{2,3}$ \\ Wanhui $\operatorname{Liu}^{2,3}$ \\ Youxin $\mathrm{Li}^{1-3}$ \\ 'College of Life Science, Jilin \\ University, Changchun, China; ${ }^{2}$ State \\ Key Laboratory of Long-acting and \\ Targeting Drug Delivery System, Luye \\ Pharmaceutical Co, Ltd, Yantai, China; \\ ${ }^{3}$ School of Pharmacy, Yantai University, \\ Yantai, China \\ *These authors contributed equally \\ to this work
}

Correspondence: Youxin Li

Life Science Building, Jilin University,

2699 Qianjin Street, Changchun, China

Tel +8643185168648

Fax +86 43I 85I68637

Email liyouxin@jlu.edu.cn

\begin{abstract}
To improve the pharmacokinetics and stability of recombinant human erythropoietin (rhEPO), rhEPO was successfully formulated into poly(ethylene glycol)-poly(d,1-lactide) (PEGPLA) di-block copolymeric micelles at diameters ranging from 60 to $200 \mathrm{~nm}$ with narrow polydispersity indices (PDIs; PDI $<0.3$ ) and trace amount of protein aggregation. The zeta potential of the spherical micelles was in the range of -3.78 to $4.65 \mathrm{mV}$ and the highest encapsulation efficiency of rhEPO in the PEG-PLA micelles was about $80 \%$. In vitro release profiles indicated that the stability of rhEPO in the micelles was improved significantly and only a trace amount of aggregate was found. Pharmacokinetic studies in rats showed highly enhanced plasma retention time of the rhEPO-loaded PEG-PLA micelles in comparison with the native rhEPO group. Increased hemoglobin concentrations were also found in the rat study. Native polyacrylamide gel electrophoresis results demonstrated that rhEPO was successfully encapsulated into the micelles, which was stable in phosphate buffered saline with different $\mathrm{pHs}$ and concentrations of $\mathrm{NaCl}$. Therefore, PEG-PLA micelles can be a potential protein drug delivery system.
\end{abstract}

Keywords: rhEPO, PEG-PLA micelle, in vitro, pharmacokinetics, pharmacodynamics

\section{Introduction}

During the last decade, protein drug delivery has become a focus of research due to the large number of recombinant proteins being investigated for therapeutic applications. Most therapeutic proteins that are administered in the native form are susceptible to biodegradation or metabolism, have low membrane permeability, or can be rapidly eliminated from the blood circulation. Recombinant human erythropoietin (rhEPO), a glycoprotein with 165 amino acids and about $40 \%$ carbohydrates, ${ }^{1-3}$ has been widely used for the treatment of anemia. Clinically, rhEPO was given by long-term multiple weekly injections subcutaneously or intravenously (three injections per week for years). There are several known issues associated with rhEPO-based therapeutic applications that should be strongly considered: short protein half-life in vivo; side effects caused by multiple or high doses administered; and possible aggregation of the protein during manipulation, leading to adverse antibody response which may convert the anemia curer to anemia causer. ${ }^{4}$ Therefore, it is crucial to develop a novel formulation of rhEPO with sustained release, less aggregation, and improvements in patient compliance and therapeutic efficiency.

PEGylation can stabilize rhEPO, reduce renal excretion, and prolong blood circulation, ${ }^{5}$ but pharmacological activity is reduced. ${ }^{6,7}$ rhEPO has also been investigated in the formulations of microspheres, gelatin hydrogels, microneedles, and gastro-intestinal patches to resolve the problems, ${ }^{8-12}$ but with limited progress. submit your manuscript $\mid$ www.dovepress.com

Dovepress

http://dx.doi.org// 0.2147/IJN.S38648 
Nanoscale vehicles have also been used as a versatile carrier for the delivery of therapeutic proteins. ${ }^{13,14}$ Polymeric micelles have recently received considerable attention as a promising nanoscale delivery system. ${ }^{15,16}$ Polymeric micelles consist of a hydrophobic inner core surrounded by a hydrophilic outer shell, which are easy to prepare and can incorporate various drugs into their inner cores with relatively high stability by chemical conjugation or physical entrapment. ${ }^{17-19}$ Polyethylene glycol (PEG), which is the most popular hydrophilic outer shell, has been used as a source of hydrophilicity due to its outstanding physicochemical and biological properties, including solubility in water and organic solvents, non-toxicity, and providing a stabilizing interface between the micelle core and the aqueous phase. In addition, PEG is able to avoid protein adsorption and subsequent non-specific uptake by the reticuloendothelial system following intravenous injection. ${ }^{20-22} \mathrm{As}$ one of the most prominent biodegradable polymers, polylactic acid (PLA) is widely used in the fields of protein delivery and tissue engineering due to its non-toxic, biocompatible, and biodegradable characteristics. ${ }^{23-26}$ PEG-PLA micelles have been used as carriers of hydrophobic compounds to increase solubility and loading capability. ${ }^{17}$ However, only a few studies on proteins encapsulated in PEG-PLA micelles have been reported up to date.

In the present study, rhEPO was encapsulated into PEGPLA micelles to extend its plasma half-life $\left(\mathrm{t}_{1 / 2}\right)$ and increase its area under curve (AUC) and efficacy. In vitro and in vivo studies were carried out and the results showed strong promise for use of micelles to encapsulate proteins.

\section{Materials and methods Materials}

The rhEPO was purchased from Shenzhen Xinpeng Bioengineering Co, Ltd (Shenzhen, China). Methoxypolyethylene glycol $\left(M_{n}=2000,5000,10,000 \mathrm{~g} / \mathrm{mol}\right)$ was purchased from Aladdin Chemistry Co, Ltd (Shanghai, China). D, L-lactide was obtained from Purac Biomaterials (Shanghai, China). A human erythropoietin enzyme-linked immunosorbent assay (ELISA) kit was purchased from R\&D Systems (Minneapolis, MN, USA). All other chemicals used were of analytical grade.

The experiments were performed on female SpragueDawley (SD) rats weighing $250 \pm 20 \mathrm{~g}$. The animals were kept in plastic cages in a room maintained at $20^{\circ} \mathrm{C} \pm 4^{\circ} \mathrm{C}$, with a 12:12 light-dark cycle. Food and water were freely available. All experiments were performed in strict accordance with the Guide for the Care and Use of Laboratory Animals as adopted by the China National Institutes of Health.

\section{Synthesis and characterization of PEG-PLAs}

Synthesis of PEG-PLAs

Different amounts of D, L-lactide were mixed with pre-dried PEG $\left(M_{n}=2000,5000,10,000 \mathrm{~g} / \mathrm{mol}\right)$ under nitrogen. Anhydrous toluene was added and followed by stannous octoate $[0.1 \%(\mathrm{w} / \mathrm{w})$, in toluene] as a catalyst. The mixture was heated to $120^{\circ} \mathrm{C}$ and refluxed for 24 hours. All reactions were carried out under an environment free of oxygen and moisture. The products were purified by repeated dissolution into dichloromethane and precipitated by cold ethyl ether. The isolated polymer was vacuum dried at $25^{\circ} \mathrm{C}$.

\section{Characterization of PEG-PLAs}

The structure of the copolymer was analyzed by ${ }^{1} \mathrm{H}$ NMR (Bruker ARX-400; Bruker Spectrospin Inc, Zurich, Switzerland). The molecular weight and the polydispersity of PEG-PLA were determined by gel permeation chromatography. The thermo-properties of the copolymers were studied by differential scanning calorimetry (DSC821E; Mettler Toledo, Greifensee, Switzerland). The characteristics of the block copolymer PEG-PLAs were listed in Table 1.

\section{Preparation and characterization of micelles PEG-PLA micelles preparation}

The solvent evaporation method was chosen to construct PEG-PLA micelles. First, the appropriate amount of PEG-PLA was dissolved in $1 \mathrm{~mL}$ of acetone. Then the PEG-PLA/acetone solution and rhEPO solution $(50,000 \mathrm{IU} / \mathrm{mL}, 400 \mu \mathrm{L})$ were added dropwise into $10 \mathrm{~mL}$ phosphate buffered saline (PBS; $10 \mathrm{mM}$, different $\mathrm{pHs}$ ) under stirring (Magnetism Msier 85-2B; Jingtan Medical Instrument Factory, Jiangsu, China), and the theoretical drug loading was $1 \%$. After evaporation for 3 hours, the $\mathrm{pH}$ value of the product was adjusted to 7.4 , before being passed through a $0.45 \mu \mathrm{m}$ filter. Finally, the micelles were obtained and stored at $4^{\circ} \mathrm{C}$ for further use. Blank PEGPLA micelles were prepared without rhEPO by the same method as described above. The preparation conditions for the micelles are shown in Table 2.

\section{Critical micelle concentration (CMC)}

The CMC was determined by the fluorescence spectroscopy method. ${ }^{27}$ First, blank PEG-PLA micelles were prepared as described above. Ten milligrams of PEG-PLA dissolved in $1 \mathrm{~mL}$ of acetone was added dropwise to $10 \mathrm{~mL}$ 
Table I Characteristics of PEG-PLA synthesized polymers

\begin{tabular}{|c|c|c|c|c|c|c|c|c|}
\hline \multirow[t]{2}{*}{ Batch } & \multirow{2}{*}{$\begin{array}{l}\text { Copolymer weight ratio } \\
M_{\mathrm{nPEG}} / \mathrm{M}_{\mathrm{nPLA}}{ }^{\mathrm{a}}\end{array}$} & \multirow[t]{2}{*}{ EG/LA ${ }^{b}$} & \multicolumn{3}{|l|}{ GPC } & \multicolumn{2}{|l|}{ DSC } & \multirow{2}{*}{$\begin{array}{l}\text { CMC } \\
(\mathrm{mol} / \mathrm{L})\end{array}$} \\
\hline & & & $M_{n}(k D a)$ & $M_{w}(k D a)$ & $M_{w} / M_{n}$ & $\mathrm{~T}_{\mathrm{m}}\left({ }^{\circ} \mathrm{C}\right)$ & $\Delta \mathbf{H}_{\mathrm{m}}(\mathrm{J} / \mathrm{g})$ & \\
\hline I & $5000: 3900$ & 2.1 & 14.16 & 15.48 & 1.07 & 53.81 & -85.13 & $1.8 \times 10^{-8}$ \\
\hline 2 & $5000: 5800$ & 1.4 & 15.89 & 17.00 & 1.09 & 51.96 & -56.97 & $1.3 \times 10^{-8}$ \\
\hline 3 & $5000: 7600$ & 1.1 & 17.55 & 23.06 & 1.36 & 53.03 & -48.36 & $1.1 \times 10^{-8}$ \\
\hline 4 & $2000: 3800$ & 0.9 & 6.16 & 8.19 & 1.32 & 54.13 & -49.86 & $1.6 \times 10^{-8}$ \\
\hline 5 & $10000: 3600$ & 4.5 & 19.24 & 21.01 & 1.09 & 63.16 & -105.35 & $5.2 \times 10^{-8}$ \\
\hline
\end{tabular}

Notes: a Copolymer weight ratio of PEG and PLA calculated from 'H NMR; ' ${ }^{2}$ molar ratio of PEG to PLA in the copolymers, calculated from 'H NMR spectra.

Abbreviations: PEG-PLA, poly(ethylene glycol)-poly(d,I-lactide); GPC, gel permeation chromatography; DSC, differential scanning calorimetry; EG/LA, Poly(ethylene glycol) /Poly(d,I-lactide); CMC, critical micelle concentration; 'H NMR, proton nuclear magnetic resonance.

PBS (10 mM, pH 7.4) with stirring and evaporated for 3 hours. The product was serially diluted to concentrations of $0.5-0.00005 \mathrm{mg} / \mathrm{mL}$. After addition of pyrene $(1 \%$ in acetone solution), the solutions were stirred at room temperature for 24 hours in the dark. The fluorescence emission ratio of $\mathrm{I}_{373 \mathrm{~nm}} / \mathrm{I}_{392 \mathrm{~nm}}$ of the solutions was recorded by a fluorescence spectrometer (RF-5301PC; Shimadzu Scientific Instruments, Kyoto, Japan) at $333 \mathrm{~nm}$ excitation wavelength. The CMC values were determined from the best-fit curves of the $\mathrm{I}_{373 \mathrm{~nm}} / \mathrm{I}_{392 \mathrm{~nm}}$ ratio against the various concentrations.

\section{Characterization of micelles}

The morphology of the PEG-PLA micelles was observed using a transmission electron microscope (TEM) (JEOL

Table 2 Preparation conditions for rhEPO-loaded PEG-PLA micelles

\begin{tabular}{|c|c|c|c|c|}
\hline Batch & PEG-PLA $^{a}$ & $\begin{array}{l}\text { PEG-PLA } \\
\text { concentration } \\
(\mathrm{mg} / \mathrm{ml})\end{array}$ & $\begin{array}{l}\text { Rotation } \\
\text { speed } \\
(\mathrm{rpm}) \\
\end{array}$ & $\begin{array}{l}\mathrm{pH} \\
\text { of PBS }\end{array}$ \\
\hline $\mathrm{I}$ & $\mathrm{PEG}_{5000}-\mathrm{PLA}{ }_{3900}$ & I & 1300 & 7.4 \\
\hline 2 & $\mathrm{PEG}_{5000}-\mathrm{PLA}_{5800}$ & I & 1800 & 7.4 \\
\hline 3 & $\mathrm{PEG}_{5000}-\mathrm{PLA}_{7600}$ & I & 1800 & 7.4 \\
\hline 4 & $\mathrm{PEG}_{5000}-\mathrm{PLA}_{5800}$ & I & 1000 & 7.4 \\
\hline 5 & $\mathrm{PEG}_{5000}-\mathrm{PLA}_{5800}$ & I & 2300 & 7.4 \\
\hline 6 & $\mathrm{PEG}_{5000}-\mathrm{PLA}_{3900}$ & 2 & 1800 & 7.4 \\
\hline 7 & $\mathrm{PEG}_{5000}-\mathrm{PLA}_{3900}$ & 3 & 1800 & 7.4 \\
\hline 8 & $\mathrm{PEG}_{5000}-\mathrm{PLA}_{3900}$ & 5 & 1800 & 7.4 \\
\hline 9 & $\mathrm{PEG}_{5000}-\mathrm{PLA}_{3900}$ & 2 & 1800 & 2.5 \\
\hline 10 & $\mathrm{PEG}_{5000}-\mathrm{PLA}_{3900}$ & 2 & 1800 & 3.6 \\
\hline II & $\mathrm{PEG}_{5000}-\mathrm{PLA}_{3900}$ & 2 & 1800 & 3.8 \\
\hline 12 & $\mathrm{PEG}_{5000}-\mathrm{PLA}_{3900}$ & 2 & 1800 & 4.0 \\
\hline 13 & $\mathrm{PEG}_{5000}-\mathrm{PLA}_{3900}$ & 2 & 1800 & 4.5 \\
\hline 14 & $\mathrm{PEG}_{5000}-\mathrm{PLA}_{3900}$ & 2 & 1800 & 7.4 \\
\hline 15 & $\mathrm{PEG}_{2000}-\mathrm{PLA}_{3800}$ & 2 & 1800 & 3.8 \\
\hline 16 & $\mathrm{PEG}_{10000}-\mathrm{PLA} \mathrm{A}_{3600}$ & 2 & 1800 & 3.8 \\
\hline
\end{tabular}

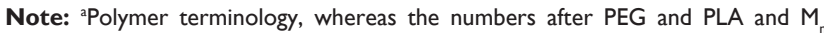
(determined by 'H NMR spectroscopy) of PEG and PLA respectively.

Abbreviations: rhEPO, recombinant human erythropoietin; PEG-PLA, poly(ethylene glycol)-poly(d,l-lactide); PBS, phosphate buffered saline.
JEM-1400; JEOL Ltd, Tokyo, Japan). The particle size, PDI, and zeta potential of the micelles were determined under room temperature by a dynamic light scattering (DLS) instrument (Nicomp 380 ZLS; PSS, Santa Barbara, CA, USA) operating at $633 \mathrm{~nm}$. The light scattering angle was set at 90 degrees, Sartorius Stedim Biotech, Goettingen, Germany.

\section{Evaluation of rhEPO entrapment and aggregation}

rhEPO-loaded PEG-PLA micelle solution (1 mL) was disintegrated completely by acetone and then extracted with PBS to obtain the total concentration of rhEPO. The micelle solution was ultrafiltrated using Vivaspin ultrafiltration spin columns (molecular weight cutoff $=100 \mathrm{kDa}$ ) (Sartorius Stedim Biotech, Goettingen, Germany) and centrifuged at a rotational speed of $3500 \mathrm{rpm}$ for 30 minutes at $4{ }^{\circ} \mathrm{C}$ (Sorvall Biofuge Primo R; Thermo Fisher Scientific, Waltham, MA, USA). The supernatant was washed with water to remove free rhEPO, then disintegrated and extracted by the same method as described above to determine the content of the encapsulated rhEPO. The aggregation of rhEPO was quantitated by size-exclusion high performance liquid chromatography (SEC-HPLC) using bovine serum albumin (BSA) as a standard (the retention time of BSA was 8.49 minutes, almost the same as the rhEPO aggregation product of 8.36 minutes). The concentrations of rhEPO and BSA were determined by an Agilent 1260 HPLC system equipped with a TSK-GEL G3000SWXL size-exclusion column and a TSK SWXL guard column (Tosoh Co, Tokyo, Japan). PBS (50 mM PBS, $300 \mathrm{mM} \mathrm{NaCl,} \mathrm{pH} \mathrm{7.4)} \mathrm{was} \mathrm{used}$ in the mobile phase, running at the rate of $0.7 \mathrm{~mL} / \mathrm{minute}$. Excitation and emission wavelengths were $280 \mathrm{~nm}$ and $340 \mathrm{~nm}$, respectively. The encapsulation efficiency (EE) was calculated according to the equation:

$$
\begin{aligned}
\mathrm{EE}(\%)= & \text { the rhEPO in supernatant } / \text { the total } \mathrm{rhEPO} \\
& \text { before ultrafiltration } \times 100 \%
\end{aligned}
$$




\section{In vitro rhEPO release}

After the free rhEPO was removed, the rhEPO-loaded micelles were evaluated in vitro and in vivo. rhEPO-loaded PEG-PLA micelles or an equivalent amount of native rhEPO solution in a dialysis bag (Spectra/Por CE Biotech Membrane; Spectrum Laboratories, Inc, Rancho Dominguez, CA, USA) (molecular weight cutoff $=300 \mathrm{kDa}$ ) was directly immersed into $100 \mathrm{~mL}$ of PBS (100 mM, pH 7.4). Dialyzate (5 mL) was withdrawn and replaced with an equivalent volume of fresh PBS at different time points of $0.25,0.5,1,2,4,6,8,10$, and 24 hours. The concentrations of free rhEPO released and residual aggregation of rhEPO recovered from the dialysis bag were assayed by SEC-HPLC in the same method as described above.

\section{Pharmacokinetic study of rhEPO-loaded PEG-PLA micelles}

SD rats (230-270 g, female), five of each group, were administrated intravenously with $2000 \mathrm{IU} / \mathrm{kg}$ of rhEPO-loaded PEG-PLA micelles or an equivalent dose of native rhEPO. Blood samples $(200 \mu \mathrm{L})$ were drawn from the retro-orbital venous plexus of the rats at $0,5,15,30$, and 45 minutes, and $1,4,8$, and 12 hours after intravenous (IV) dosing. The blood samples were centrifuged and the plasma was stored at $-70^{\circ} \mathrm{C}$ until analysis. Plasma levels of rhEPO were quantitated using an ELISA kit, according to the manufacturer's instructions (MEP00, R\&D Systems).

\section{Pharmacodynamic study of rhEPO-loaded PEG-PLA micelles}

SD rats (230-270 g, female), five of each group, were administrated intravenously with $2000 \mathrm{IU} / \mathrm{kg}$ of rhEPO-loaded PEG-PLA micelles or an equivalent dose of native rhEPO and PBS as a control. Blood samples $(200 \mu \mathrm{L})$ were drawn from the retro-orbital venous plexus of the rats at $0,1,2,3$, and 4 days after IV dosing. The blood samples were mixed with a dilution buffer provided by the manufacturer immediately, and hemoglobin (HGB) levels were determined with an automated blood analyzer (Hemavet 950; Drew Scientific, Oxford, CT, USA).

The percentage increase of HGB concentration (compared with the control group) was used to characterize the pharmacodynamics of the rhEPO-loaded PEG-PLA micelles and the native rhEPO as reported previously. ${ }^{8}$

\section{Native-PAGE}

To demonstrate the encapsulation of rhEPO within the PEGPLA micelle, native-PAGE was conducted by a mini-gel apparatus (gel size: $83 \times 75 \times 1 \mathrm{~mm}$; model DYCZ-24DN;
Beijing Liuyi Scientific Equipment Ltd, Beijing, China) using a $12 \%$ separating gel and a $5 \%$ stacking gel. The electrophoresis was run under non-reducing conditions. Blank PEG-PLA micelles, rhEPO, rhEPO-loaded PEG-PLA micelles, a mixture of blank micelles with rhEPO, and disintegrated rhEPO-loaded PEG-PLA micelles were analyzed. Protein samples were mixed with $5 \mathrm{X}$ non-reducing loading buffer [0.1 M Tris-HCl, pH 6.8; 20\% (v/v) glycerol; 0.01\% bromophenol blue]. Gels were stained in $0.1 \%(w / v)$ R250 Coomassie Brilliant Blue and destained with a solution of $10 \%(\mathrm{v} / \mathrm{v})$ ethanol and $10 \%(\mathrm{v} / \mathrm{v})$ acetic acid.

\section{Physical stability of micelles}

For physical stability of the micelles in PBS, 2 mL of PEGPLA micelle solution was mixed with PBS $(10 \mathrm{mM})$ under different conditions (different $\mathrm{pHs}$ of 2.0, 4.5, 7.4, and 10.4; different $\mathrm{NaCl}$ concentrations of $0,80,150,300$, and $600 \mathrm{mM})$.

The micelle solution was stored at $2^{\circ} \mathrm{C}-8^{\circ} \mathrm{C}$ for 2 months to test the storage stability.

\section{Statistical analysis}

All data were reported as mean \pm standard deviation. Statistical analysis between groups was determined by oneway analysis of variance followed by Dunnett's test. A value of $P<0.05$ was considered statistically significant.

\section{Results}

\section{Characterization of PEG-PLAs}

A series of PEG-PLA copolymers was synthesized successfully in our lab. The characteristics of the copolymers are listed in Table 1. $M_{n}$ of the PEG-PLA block copolymers was in the range of 6000-19,000. Melting enthalpies decreased as the content of lactide increased, due to the reduction in crystallization of PEG in the copolymer. CMC values of the copolymers (Table 1) were well associated with hydrophobic block lengths, ${ }^{27}$ which decreased with the increase of lactide content in the copolymers. This was consistent with the data reported previously. ${ }^{28-31}$ All the CMC values were low enough $\left(0.52-1.8 \times 10^{-8} \mathrm{~mol} / \mathrm{L}\right)$ to maintain thermodynamic stability of micelles after intravenous injection which induced severe dilution. ${ }^{32,33}$

\section{Physicochemical characterization of micelles}

Morphology of micelles

The morphology of PEG-PLA micelles were visualized by TEM. As shown in Figure 1A, the bright entities were 


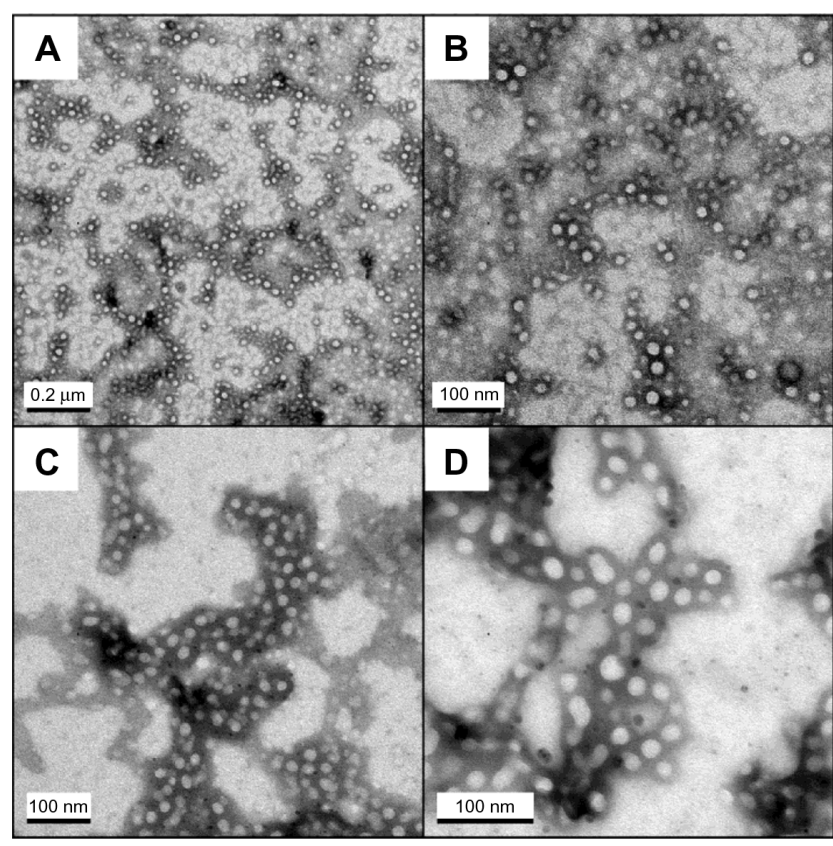

Figure I TEM images of PEG-PLA micelles. (A) PEG ${ }_{5000}-\mathrm{PLA}_{3900}$ blank micelle (B) rhEPO-loaded PEG $2000-\mathrm{PLA}_{3800}$ micelle. (C) rhEPO-loaded $\mathrm{PEG}_{5000}-\mathrm{PLA}_{3900}$ micelle. (D) rhEPO-loaded PEG ${ }_{10,000}-\mathrm{PLA}_{3600}$ micelle.

Abbreviations: TEM, transmission electron microscopy; PEG-PLA, poly(ethylene glycol)-poly(d,l-lactide).

surrounded by dark staining, which clearly indicated the presence of spherical particulates. The micelles were discrete; fairly uniform in size. Additionally, the $\mathrm{PEG}_{10,000}-\mathrm{PLA}_{3600}$ micelles showed a larger size (Figure 1D) than the other two micelles (Figure 1B and C).

\section{The particle size and zeta potential of micelles}

Particle size and zeta potential were main factors for in vivo circulation of nanoparticles. ${ }^{34}$ The PEG-PLA micelles were characterized by DLS in terms of mean size, PDI, and zeta potential (Table 3 ). The zeta potential of micelles was found to be neutral in the range of $-3.78 \mathrm{mV}$ to $4.65 \mathrm{mV}$. Micelles had a size in the range of $60-200 \mathrm{~nm}$ with a PDI between 0.15 and 0.31 suggesting good polydispersity. The increase in either block length of PLA or that of PEG when the other block was kept unchanged increased the size of the micelle. The size of $\mathrm{PEG}_{5000}-\mathrm{PLA}_{3900}$ micelles $\left(73.4 \mathrm{~nm}\right.$ ) was smaller than that of $\mathrm{PEG}_{5000}-\mathrm{PLA}_{7600}$ and $\mathrm{PEG}_{10000}-\mathrm{PLA}_{3600}$ micelles. The effect on the size and PDI of PEG-PLA micelles with respect to stirring speed, polymer concentration, and $\mathrm{pH}$ value of PBS were also studied. At a lower stirring speed of $1000 \mathrm{rpm}$, the size of the micelle was $196.4 \mathrm{~nm}$, and it was reduced to $137.5 \mathrm{~nm}$ at a stirring speed of $1800 \mathrm{rpm}$, but the size increased to $187.6 \mathrm{~nm}$ when the stirring speed was accelerated to $2300 \mathrm{rpm}$. The size also
Table 3 Characteristics of rhEPO-loaded PEG-PLA micelles

\begin{tabular}{lrrrrl}
\hline Batch & $\begin{array}{l}\text { Particle } \\
\text { size (nm) }\end{array}$ & PDI & \multicolumn{1}{l}{$\begin{array}{l}\text { Zeta } \\
\text { potential } \\
(\mathbf{m V})\end{array}$} & EE (\%) & \multicolumn{1}{l}{$\begin{array}{l}\text { Aggregation } \\
(\%)\end{array}$} \\
\hline 1 & $73.4 \pm 3.6$ & 0.23 & $-3.78 \pm 2.12$ & $8.2 \pm 2.1$ & $5.16 \pm 1.03$ \\
2 & $137.5 \pm 5.3$ & 0.22 & $4.65 \pm 1.65$ & $7.4 \pm 3.4$ & $3.35 \pm 1.52$ \\
3 & $177.8 \pm 6.2$ & 0.31 & $4.55 \pm 1.38$ & $3.1 \pm 1.2$ & $23.02 \pm 4.37$ \\
4 & $196.4 \pm 4.9$ & 0.22 & $1.14 \pm 1.65$ & $10.4 \pm 3.1$ & $4.70 \pm 1.46$ \\
5 & $187.6 \pm 3.2$ & 0.25 & $-3.06 \pm 2.39$ & $3.3 \pm 1.2$ & $3.68 \pm 1.21$ \\
6 & $80.4 \pm 1.6$ & 0.22 & $0.68 \pm 1.26$ & $14.7 \pm 32$ & Trace* \\
7 & $83.4 \pm 3.1$ & 0.27 & $0.92 \pm 1.53$ & $5.2 \pm 1.6$ & $3.38 \pm 0.95$ \\
8 & $77.4 \pm 1.6$ & 0.43 & $0.43 \pm 4.29$ & $\mathrm{~N}$ & $\mathrm{~N}$ \\
9 & $67.2 \pm 2.6$ & 0.19 & $-0.46 \pm 1.14$ & $47.2 \pm 35$ & \multicolumn{1}{l}{ Trace* } \\
10 & $70.1 \pm 1.7$ & 0.21 & $-0.75 \pm 0.96$ & $57.5 \pm 4.3$ & Trace* \\
11 & $71.5 \pm 2.3$ & 0.15 & $0.21 \pm 1.32$ & $71.5 \pm 1.6$ & \multicolumn{1}{l}{ Trace* } \\
12 & $723 \pm 1.5$ & 0.19 & $-0.54 \pm 0.95$ & $58.3 \pm 1.8$ & Trace* \\
13 & $69.8 \pm 1.8$ & 0.25 & $1.86 \pm 0.91$ & $60.4 \pm 4.6$ & Trace* \\
14 & $80.2 \pm 2.1$ & 0.23 & $-1.35 \pm 1.46$ & $20.6 \pm 3.4$ & Trace* \\
15 & $73.4 \pm 2.8$ & 0.24 & $1.28 \pm 0.98$ & $54.9 \pm 3.2$ & Trace* \\
16 & $131.2 \pm 4.3$ & 0.28 & $-0.76 \pm 1.23$ & $79.2 \pm 4.9$ & Trace* \\
\hline
\end{tabular}

Notes: *Trace meant below the limit of detection. $\mathrm{N}$ demonstrated didn't detect. Abbreviations: rhEPO, recombinant human erythropoietin; PEG-PLA, poly(ethylene glycol)-poly(d,I-lactide); PDI, polydispersity index; EE, encapsulation efficiency.

increased slightly with increased PEG-PLA concentration. There was no significant difference in size of the micelles prepared at different $\mathrm{pHs}$.

\section{EE of micelles and aggregation of rhEPO}

The EE and percentage of the protein aggregation of rhEPOloaded PEG-PLA micelles were investigated (Table 3). Figure 2 shows that $\mathrm{pH}$ played a pivotal role in the EE. At $\mathrm{pH} 7.4$, the EE of the optimized PEG-PLA micelle was 21\% with trace aggregation of rhEPO (Batch 14). A significant

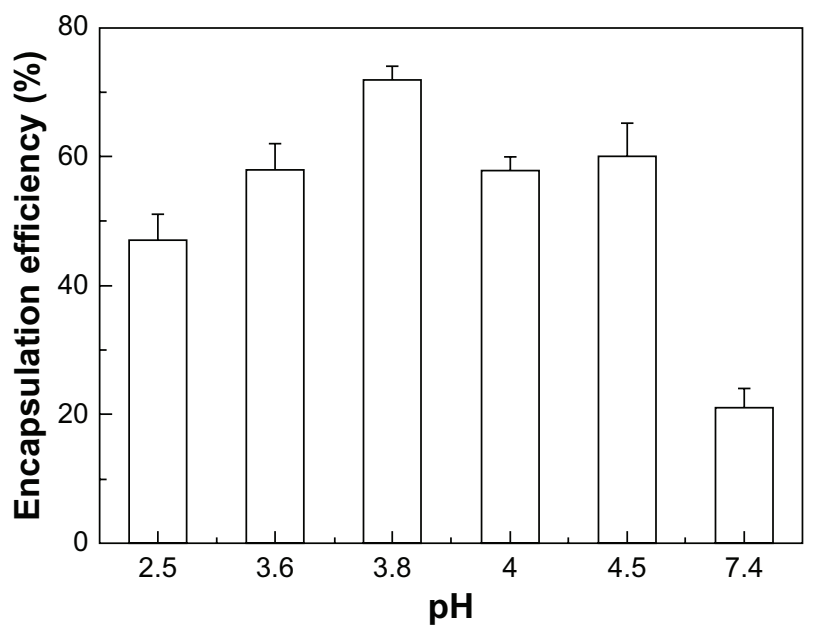

Figure 2 The encapsulation efficiency of the PEG-PLA micelles prepared at different $\mathrm{pHs}$.

Abbreviation: PEG-PLA, poly(ethylene glycol)-poly(d,I-lactide). 
improvement of EE was found when the $\mathrm{pH}$ was in the range of 3.5-4.5 and the highest EE of 79.2\% was obtained at $\mathrm{pH} 3.8$ with only a trace amount of aggregate. The EE increased with lengthening of the PEG block; eg, the EE of $\mathrm{PEG}_{10,000}-\mathrm{PLA}_{3600}$ micelle was about $20 \%$ higher than that of $\mathrm{PEG}_{2000}-\mathrm{PLA}_{3800}$ micelle (Table 3).

\section{In vitro release}

Figure 3 shows the in vitro cumulative release of rhEPO from the micelles with different PEG-PLAs compared to that of the native rhEPO solution. The cumulative release of rhEPO from all micelles reached almost $80 \%$, while native rhEPO was only $50 \%$. The $\mathrm{PEG}_{5000}-\mathrm{PLA}_{5800,7600}$ micelles weren't evaluated in vitro in our study due to their larger size (137.5, $177.8 \mathrm{~nm}$ ). It was previously reported that nanoparticles with larger size $(>100 \mathrm{~nm})$ were cleared quickly in blood. ${ }^{35}$ The micelles of batch 11,15 , and 16 (Table 3 ) were carried out in vitro to investigate the effect of block length of PEG. The results demonstrated that $\mathrm{PEG}_{5000}-\mathrm{PLA}_{3900}$ micelles released slower than the other two micelles. After the release of rhEPO in vitro, the amount of aggregated rhEPO in the dialysis bag was assayed in both the $\mathrm{PEG}_{5000}-\mathrm{PLA}_{3900}$ micelle group and the native rhEPO group. About 30\% less aggregation was found in the $\mathrm{PEG}_{5000}-\mathrm{PLA}_{3900}$ micelle group indicating that the stability of rhEPO was improved significantly in the micelle (Figure 4).

\section{Pharmacokinetic study}

Circulating levels of rhEPO were measured by ELISA following IV injection in rats. The mean plasma concentrationtime curves are shown in Figure 5. The pharmacokinetic parameters were calculated by the pharmacokinetic software

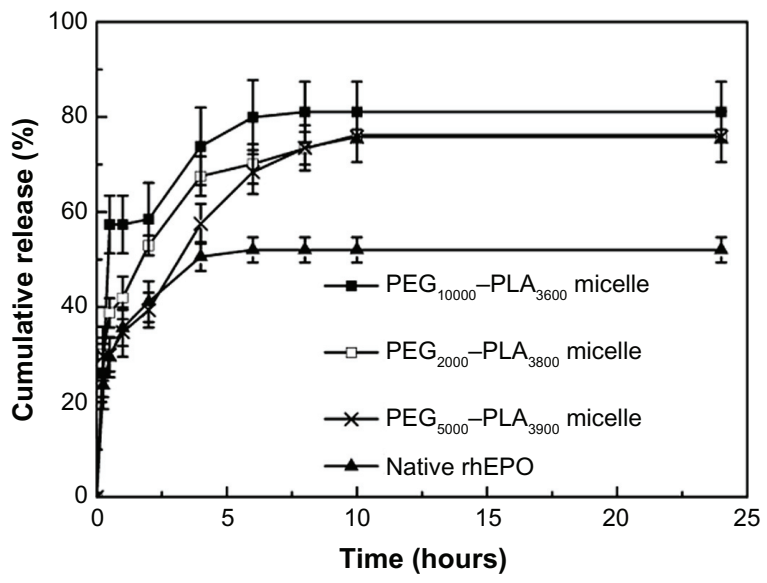

Figure 3 In vitro release behaviors of rhEPO-loaded PEG-PLA micelles (Table 2, batch 11,15 , and 16$)$ in PBS $(n=3)$.

Abbreviations: PEG-PLA, poly(ethylene glycol)-poly(d,l-lactide); rhEPO, recombinant human erythropoietin; PBS, phosphate buffered saline.
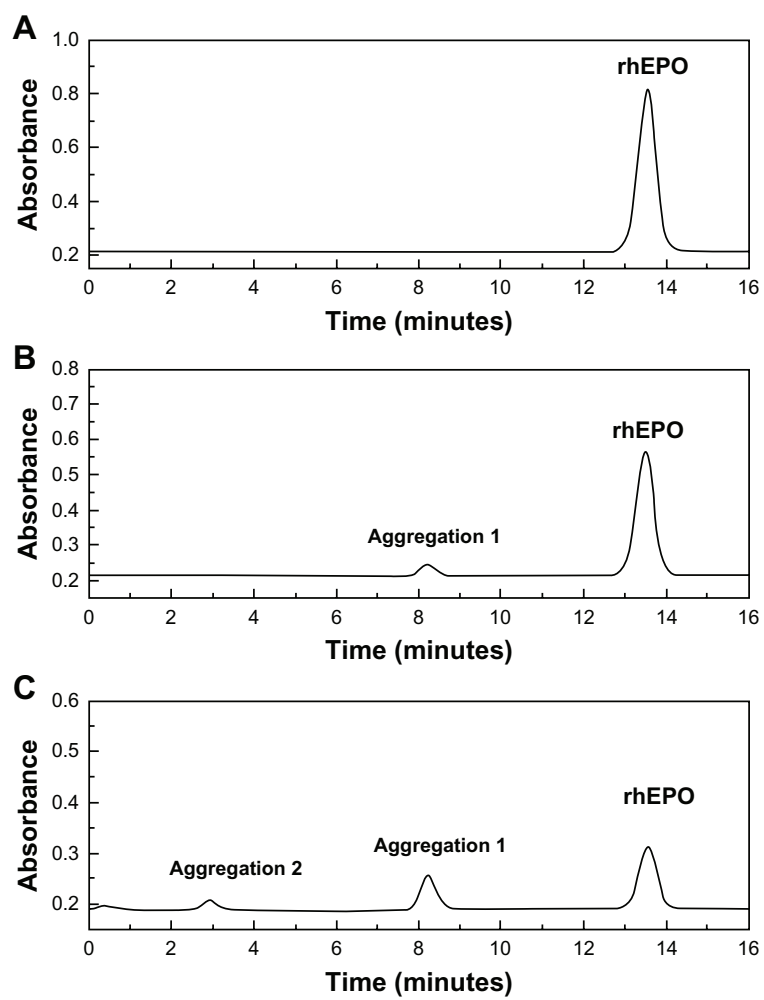

Figure 4 SEC-HPLC chromatograms of rhEPO. (A) Native rhEPO solution; (B) RhEPO recovered from the dialysis bag after in vitro release of rhEPO-loaded $\mathrm{PEG}_{5000} \mathrm{PLA}_{3900}$ micelle; (C) RhEPO recovered from the dialysis bag after in vitro release of native rhEPO solution.

Abbreviations: SEC-HPLC, size-exclusion high performance liquid chromatography; rhEPO, recombinant human erythropoietin; PEG-PLA, poly(ethylene glycol)poly(d,I-lactide).

DAS 2.1.1 (Drug And Statistics; a computer program produced by the Clinical Trial Center of Shanghai University of Traditional Chinese Medicine, Shanghai, China) and summarized in Table 4. $\mathrm{C}_{\max }$ of the $\mathrm{PEG}_{2000}-\mathrm{PLA}_{3800}$ and $\mathrm{PEG}_{5000}-$ $\mathrm{PLA}_{3900}$ micelle groups were 32.83 and $39.88 \mathrm{ng} / \mathrm{mL}$, respectively, much higher than that of the native rhEPO group $(12.07 \mathrm{ng} / \mathrm{mL})(P<0.05)$. Plasma half-lives of the two micelle groups were also prolonged compared to that of the native rhEPO group (4.36 or 4.12 hours vs 2.02 hours, $P<0.05)$. Though a longer PEG block reduced the improvement of $\mathrm{C}_{\max }$, the $\mathrm{C}_{\max }(16.65 \mathrm{ng} / \mathrm{mL})$ of the $\mathrm{PEG}_{10,000}-\mathrm{PLA}_{3600}$ micelles was still significantly higher than that of the native rhEPO group. The $\mathrm{PEG}_{10,000}-\mathrm{PLA}_{3600}$ micelles didn't prolong $\mathrm{t}_{1 / 2}$ significantly (3.62 hours vs 2.02 hours, $P>0.05$ ). The AUC of all the micelles increased compared to that of the native rhEPO group. Moreover, a 2-fold increase in AUC was found in the $\mathrm{PEG}_{5000}-\mathrm{PLA}_{3900}$ micelle group.

\section{Pharmacodynamic study}

HGB concentration changes of the rats after the injections of the micelles, native rhEPO (2000 IU/kg), and PBS were 


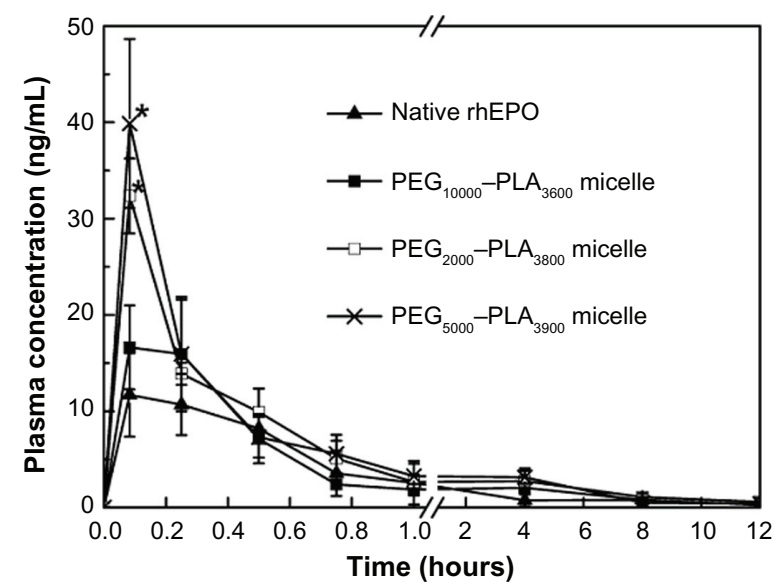

Figure 5 Plasma concentration versus time curve for rhEPO after IV administration of rhEPO-loaded PEG-PLA micelles (Table 2, batch II, I5, and 16) (2000 IU/kg). Notes: Each point represents mean $\pm \operatorname{SD}(n=5)$; $* P<0.05$ (micelle group vs native rhEPO group).

Abbreviations: rhEPO, recombinant human erythropoietin; IV, intravenous; PEGPLA, poly(ethylene glycol)-poly(d,-l-lactide); SD, standard deviation.

determined by automated blood analyzer (Figure 6). On the second day after IV administration, the HGB level started to increase. In the case of the $\mathrm{PEG}_{5000}-\mathrm{PLA}_{3900}$ and $\mathrm{PEG}_{10,000}$ $\mathrm{PLA}_{3600}$ micelles, the percentage increase of HGB concentration was enhanced to $43.45 \%$ and $48.72 \%$, respectively, much higher $(P<0.05)$ than that of the native rhEPO group (25.59\%). The percentage increase of HGB concentration of $38.43 \%$ in the case of the $\mathrm{PEG}_{2000}-\mathrm{PLA}_{3800}$ micelles indicated that a short PEG block might reduce the improvement lightly. On the third day, the percentage increase of $\mathrm{HGB}$ concentration of the $\mathrm{PEG}_{2000}-\mathrm{PLA}_{3800}$ and $\mathrm{PEG}_{5000}-\mathrm{PLA}_{3900}$ micelles were at $48.21 \%$ and $48.71 \%$, significantly higher than that of the native rhEPO group $(22.49 \%, P<0.05)$. In the case of the $\mathrm{PEG}_{10,000}-\mathrm{PLA}_{3600}$ micelles, the percentage increase of HGB concentration (34.04\%) on the third day was reduced from $48.72 \%$ achieved on the second day, still higher than the $22.48 \%$ increase of the native rhEPO group.

Table 4 Pharmacokinetic parameters of rhEPO in the SpragueDawley rats

\begin{tabular}{|c|c|c|c|c|}
\hline \multirow[t]{2}{*}{ Parameters } & \multirow{2}{*}{$\frac{\text { AUC }}{\mu \mathrm{g} / \mathrm{L} \cdot \mathrm{h}}$} & \multirow{2}{*}{$\frac{T_{1 / 2}}{h}$} & \multirow{2}{*}{$\frac{\mathrm{C}_{\max }}{\mathrm{ng} / \mathrm{mL}}$} & \multirow[t]{2}{*}{ Ratio* } \\
\hline & & & & \\
\hline Native rhEPO & $15.96 \pm 8.02$ & $2.02 \pm 0.59$ & $12.07 \pm 4.33$ & 1.00 \\
\hline $\begin{array}{l}\text { PEG }_{10000^{-}} \\
\text {PLA }_{3600} \text { micelle }\end{array}$ & $23.01 \pm 2.56$ & $3.62 \pm 0.09$ & $16.65 \pm 4.35$ & $\mathrm{I} .44$ \\
\hline $\begin{array}{l}\text { PEG }_{5000}- \\
\text { PLA }_{3900} \text { micelle }\end{array}$ & $32.83 \pm 3.87$ & $4.36 \pm 0.36$ & $39.88 \pm 8.76$ & 2.00 \\
\hline $\begin{array}{l}\mathrm{PEG}_{2000}- \\
\mathrm{PLA}_{3800} \text { micelle }\end{array}$ & $31.75 \pm 6.53$ & $4.12 \pm 0.41$ & $32.36 \pm 3.89$ & 1.99 \\
\hline
\end{tabular}

Note: $*$ Ratio $=($ AUC of rhEPO-loaded PEG-PLA micelles $) /(A \cup C$ of native rhEPO).

Abbreviations: rhEPO, recombinant human erythropoietin; AUC, area under curve; ${ }_{\text {TI/2}}$, plasma half-life; PEG-PLA, poly(ethylene glycol)-poly(d,I-lactide).

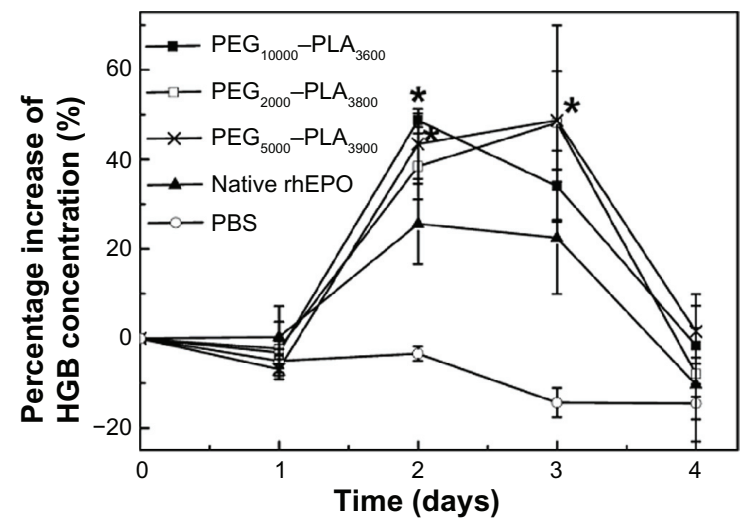

Figure 6 Percentage increase of hemoglobin concentration of the rats after IV administration of rhEPO-loaded PEG-PLA micelles (Table 2, batch II, 15, and I6), native rhEPO and PBS (2000 IU/kg).

Notes: Each point represents mean $\pm S D(n=5) ; * P<0.05$ (micelle group vs native rhEPO group).

Abbreviations: IV, intravenous; rhEPO, recombinant human erythropoietin; PEG-PLA, poly(ethylene glycol)-poly(d,I-lactide); PBS, phosphate buffered saline; $\mathrm{SD}$, standard deviation.

The significantly higher HGB concentrations of all the micelles were maintained until the fourth day.

\section{Native-PAGE}

No protein bands in the lanes of blank PEG-PLA micelles and rhEPO-loaded PEG-PLA micelles were observed (Figure 7), which could be found in the lanes of native rhEPO, the mixture of blank micelles with rhEPO, and the disintegrated rhEPO-loaded PEG-PLA micelle.

\section{Physical stability of micelles}

Particle size and PDI were the main characteristics we used to measure the physical stability of the micelles. A 2-month

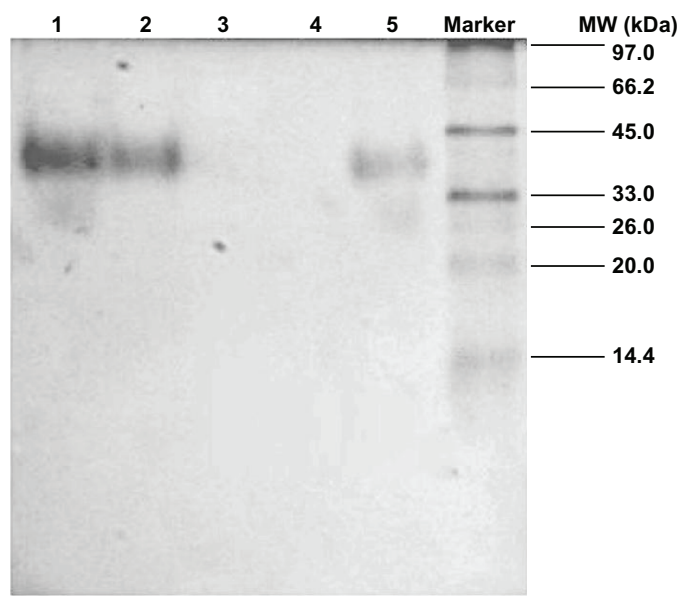

Figure 7 Native-PAGE analysis.

Notes: Lanes: I, native rhEPO solution; 2, the mixture of blank PEG-PLA micelle with rhEPO; 3, blank PEG-PLA micelle; 4, rhEPO-loaded PEG-PLA micelle; 5, rhEPO-loaded PEG-PLA micelle destroyed by acetone.

Abbreviations: rhEPO, recombinant human erythropoietin; PEG-PLA, poly(ethylene glycol)-poly(d,l-lactide). 
stability test of the PEG-PLA micelles at $2^{\circ} \mathrm{C}-8^{\circ} \mathrm{C}$ was carried out (Table 5). The particle size of the micelles kept almost the same and PDI was lower than 0.3 until 1 month, which showed that the micelle was stable within 1 month.

The micelle was stable in a wide range of $\mathrm{pH}(2.0-10.4$, data not shown). The size of the micelles was not changed with $\mathrm{NaCl}$ concentration up to $300 \mathrm{mM}$ (Figure 8), but when the concentration of $\mathrm{NaCl}$ was increased to $600 \mathrm{mM}$, the particle size started to increase, indicating aggregation of micelles.

\section{Discussion}

This study was designed to investigate the effect of encapsulation of rhEPO in polymeric micelles on in vitro release, pharmacokinetics, and efficacy. rhEPO was successfully formulated into polymer micelles of PEG-PLA diblock copolymers with a relatively high EE. Results showed the polymeric micelles increased AUC, prolonged $t_{1 / 2}$, and enhanced drug efficacy.

The DLS method was used to analyze the particle size and zeta potential of the micelles. The results demonstrated that the PEG-PLA micelles showed a growing size with the increase of lactide content in the polymer due to interfacial tension between the polymer solution and the aqueous phase. ${ }^{36}$ The mean size of the PEG ${ }_{10,000}-\mathrm{PLA}_{3600}$ micelles (131.2 nm) was larger than that of the $\mathrm{PEG}_{5000}-\mathrm{PLA}_{3900}$ micelles, due to a larger corona thickness of the micelle with the increase of PEG block length in the PEG-PLA. For example, the corona thickness of PEG $\left(M_{w}=12,000\right)$ in the micelles was 16.7-26.2 nm. ${ }^{37}$ The particle size of the PEG-PLA micelles was also influenced by stirring speed. The balance between the mutual attraction of hydrophobic segments and repulsion of hydrophilic segments to formulate micelles was influenced by the existence of stirring to a certain extent, leading to an increase of the energy in the system. In order to decrease the interfacial energy, the particle size of the micelles was increased. ${ }^{38}$ Thus, at a higher stirring speed of $2300 \mathrm{rpm}$, the mean particle size of the micelles was $50 \mathrm{~nm}$ larger than that prepared at $1800 \mathrm{rpm}$. Due to influences of the dehydration and collapse of the hydrophilic PEG corona of the micelles, TEM (Figure 1) showed much smaller particle size than that determined by DLS. ${ }^{39}$

Table 5 The stability of the micelle (Table 2 , batch II) at $2^{\circ} \mathrm{C}-8^{\circ} \mathrm{C}$

\begin{tabular}{llllllll}
\hline Time (day) & $\mathbf{0}$ & $\mathbf{I}$ & $\mathbf{3}$ & $\mathbf{7}$ & $\mathbf{1 5}$ & $\mathbf{3 0}$ & $\mathbf{6 0}$ \\
\hline Particle size $(\mathrm{nm})$ & 71.5 & 67.1 & 66.3 & 66.2 & 65.1 & 62.9 & 62.5 \\
PDI & 0.26 & 0.26 & 0.26 & 0.30 & 0.29 & 0.28 & 0.42
\end{tabular}

Abbreviation: PDI, polydispersity index.
Nanoparticles with a positive charge are easy to absorb with albumin in blood; while those with a negative charge tend to adhere to the surface of platelets. ${ }^{40}$ Due to a neutral PEG corona of the micelles in PBS at pH 7.4, the zeta potential of the micelles was close to zero, which could reduce the absorbance of the micelle with components in blood, increase the stability, and provide a longer circulation in vivo.

To remove free non-encapsulated rhEPO, a filtration/ centrifugation separation technique was applied. The particle size and PDI of the micelles were not modified by the filtration/centrifugation process, but about $50 \%$ of the free rhEPO remained adsorbed in the filter. Therefore, the ratio of rhEPO encapsulated in PEG-PLA micelles to the total rhEPO was calculated as EE. The highest EE of $79 \%$ was obtained at $\mathrm{pH} 3.8$, in which rhEPO had the lowest solubility (the isoelectric point of rhEPO is $\mathrm{pH} 3.7-4.1$ ). The hydrophilicity reduction of rhEPO at this $\mathrm{pH}$ led to more rhEPO molecules encapsulated into PEG-PLA micelles. The result of native-PAGE (Figure 7) also verified that almost all of the rhEPO molecules encapsulated into the micelles remained in their original native form.

Based on the rhEPO release curves in vitro (Figure 3), we speculated that the release of rhEPO within 0.5 hours was due to the effusion of rhEPO from the surface of the micelles. After 1 hour, a consistent and stable tendency was observed in the release curves because a constant quantity of rhEPO was exposed at the surface of the micelles owing to the constant effusion speed of the rhEPO (Figure 3). Due to an improved stability, the rhEPO released from PEG-PLA micelles in vitro was significantly more than that released from the native rhEPO group (Figure 3), which was consistent with a $30 \%$ less residual aggregation of rhEPO in the dialysis bag of the micelle group (Figure 4). PEG conjugated to PLA helped prevent protein aggregation and protein interfacial interactions with the solid surface of the container. ${ }^{41,42} \mathrm{PEG}_{10,000}-\mathrm{PLA}_{3600}$ micelles released faster than $\mathrm{PEG}_{5000}-\mathrm{PLA}_{3900}$ micelles, which might be explained due to the higher hydrophilicity of the micelles containing the longer PEG chain.

The results of rat study showed improved $t_{1 / 2}$ and AUC of the micelles compared to that of native rhEPO group (Figure 5 and Table 4). Although the main site and mechanism of the clearance of rhEPO from circulation is not fully known yet, the kidneys have been shown to play a role in its metabolic removal. ${ }^{43-45}$ Polymer nanoparticles larger than $10 \mathrm{~nm}$ in diameter could avoid first-pass renal filtration. ${ }^{46}$ Improved pharmacokinetic performances of rhEPO in the PEG-PLA micelles with a size of $60-150 \mathrm{~nm}$ were attributed to reduced renal filtration as well as the reduction of the aggregation of rhEPO in the micelles. PEG prevented the uptake and rapid 


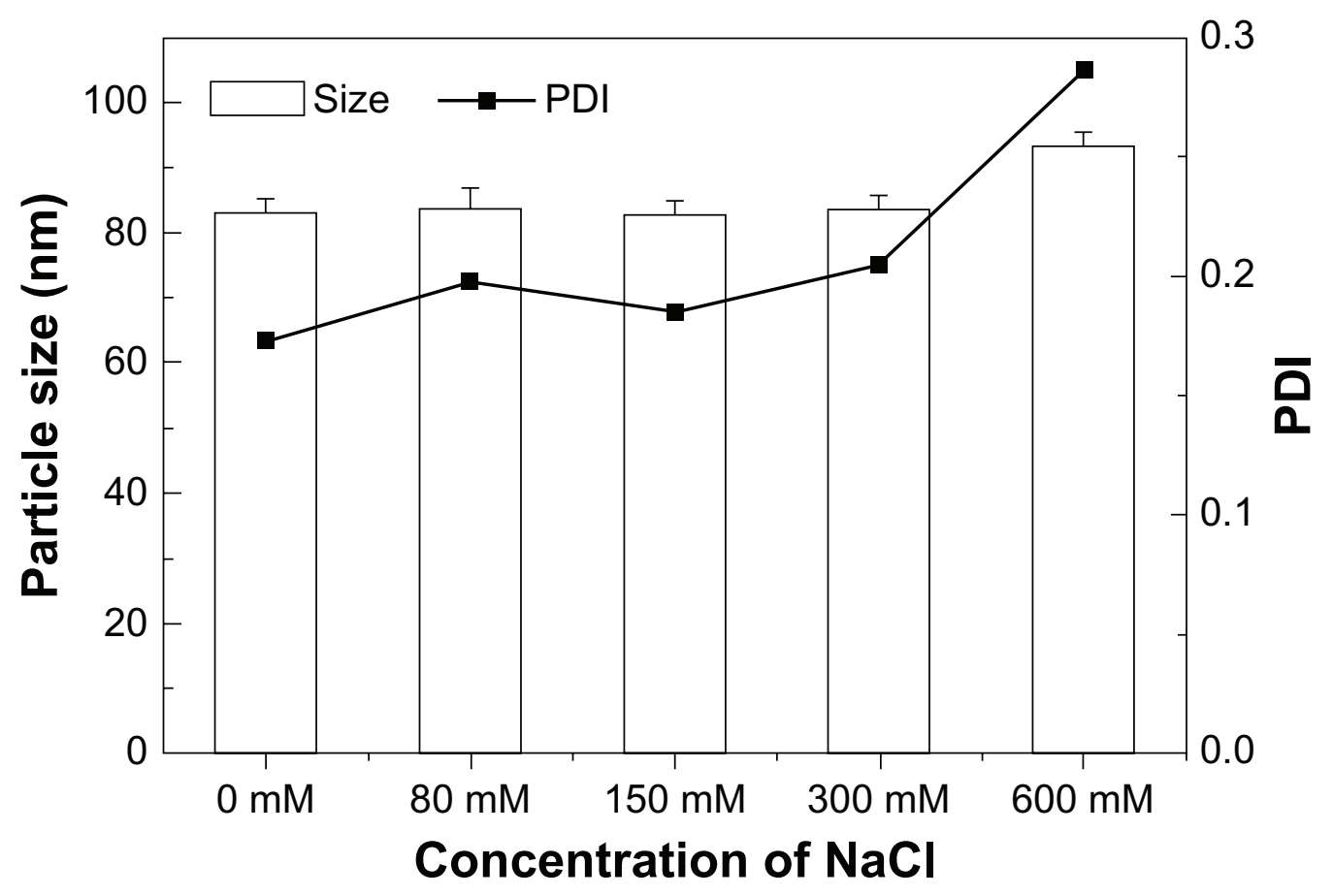

Figure 8 The mean particle size and PDI of the PEG-PLA micelle in PBS with different concentrations of $\mathrm{NaCl}$.

Abbreviations: PDI, polydispersity index; PEG-PLA, poly(ethylene glycol)-poly(d,I-lactide); PBS, phosphate buffered saline.

clearance of the micelles in vivo by the reticuloendothelial system and also prevented the interaction of the payload with proteolytic enzymes, and thus increased the AUC and prolonged the $\mathrm{t}_{1 / 2} \cdot{ }^{47}$ The $\mathrm{t}_{1 / 2}$ of the $\mathrm{PEG}_{10,000}-\mathrm{PLA}_{3600}$ micelles was shorter than the other two PEG-PLA micelles in vivo (3.62 hours vs 4.36 hours or 4.12 hours, Table 4 ), which was due to its larger particle size (131.2 nm vs $71.5 \mathrm{~nm}$ or $73.4 \mathrm{~nm})$, a main factor for the clearance in vivo. As previously reported, the blood clearance of $80 \mathrm{~nm}$ nanoparticles was half as fast as the clearance of $170 \mathrm{~nm}$ and $240 \mathrm{~nm}$ particles. ${ }^{35}$

The pharmacological activity of rhEPO relies on its specific binding on cell surface receptors. Activation of the rhEPO receptor can trigger intracellular signaling events which leads to proliferation and differentiation of the cells. ${ }^{48}$ Therefore, HGB concentration of all the rats after administration of the micelles and native rhEPO started to increase on the second day. The percentage increase of HGB concentration of all the micelles was much higher on the second and the third day as compared to that of the native rhEPO group (Figure 6). Since the $\mathrm{PEG}_{10,000}-\mathrm{PLA}_{3600}$ micelles cleared faster than the other two micelles (Table 4) in vivo, on the third day, the percentage increase of HGB concentration was relatively lower for the $\mathrm{PEG}_{10,000}-\mathrm{PLA}_{3600}$ micelles as compared to the $\mathrm{PEG}_{2000}-\mathrm{PLA}_{3800}$ or $\mathrm{PEG}_{5000}-\mathrm{PLA}_{3900}$ micelles $(34.04 \%$ vs $48.21 \%$ or $48.71 \%$ ). However, there was no significant difference in efficacy among the PEG-PLA micelles.
In summary, the studies indicated that the rhEPO was successfully encapsulated in the micelles, and the protein was maintained in its native conformation and had improved stability both in vitro and in vivo. The micelles could improve the pharmacokinetics of rhEPO significantly, and a double AUC and prolonged $t_{1 / 2}$ might benefit the patient.

\section{Conclusion}

A novel formulation of rhEPO in PEG-PLA micelles, which is spherical, highly monodispersed, and stable physically in PBS for at least 1 month, has been developed and characterized. The rhEPO has been shown to be encapsulated into PEG-PLA micelles with high efficiency, which reduces its propensity to aggregate and provides conformational stability to the rhEPO molecules, and thus increases AUC, prolongs $t_{1 / 2}$, and enhances drug efficacy. Improved stability of rhEPO in the micelles may decrease the need to administer large doses of the protein through continuous IV or subcutaneous injection administration for achieving therapeutic efficacy. Meanwhile, our research presents great promise for the use of micelles to encapsulate proteins.

\section{Acknowledgments}

This work was supported by the National Basic Research Program of China. (No 2010CB735602, No 2012CB724003). 


\section{Disclosure}

The authors report no conflicts of interest in this work.

\section{References}

1. Krantz SB. Erythropoietin. Blood. 1991;77(3):419-434.

2. Fukuda MN, Sasaki H, Lopez L, Fukuda M. Survival of recombinant erythropoietin in the circulation: the role of carbohydrates. Blood. 1989;73(1):84-89.

3. Dordal MS, Wang FF, Goldwasser E. The role of carbohydrate in erythropoietin action. Endocrinology. 1985;116(6):2293-2299.

4. Schellekens H. Immunologic mechanisms of EPO-associated pure red cell aplasia. Best Pract Res Clin Haematol. 2005;18(3):473-480.

5. Macdougall IC, Eckardt KU. Novel strategies for stimulating erythropoiesis and potential new treatments for anaemia. Lancet. 2006;368(9539): 947-953.

6. Francis GE, Fisher D, Delgado C, Malik F, Gardiner A, Neale D. PEGylation of cytokines and other therapeutic proteins and peptides: the importance of biological optimisation of coupling techniques. Int J Hematol. 1998;68(1):1-18.

7. Veronese FM, Mero A. The impact of PEGylation on biological therapies. Biodrugs. 2008;22(5):315-329.

8. He J, Feng M, Zhou X, et al. Stabilization and encapsulation of recombinant human erythropoietin into PLGA microspheres using human serum albumin as a stabilizer. Int J Pharm. 2011;416(1):69-76.

9. Geng Y, Yuan W, Wu F, Chen J, He M, Jin T. Formulating erythropoietin-loaded sustained-release PLGA microspheres without protein aggregation. J Control Release. 2008;130(3):259-265.

10. Venkatesan N, Uchino K, Amagase K, Ito Y, Shibata N, Takada K. Gastro-intestinal patch system for the delivery of erythropoietin. J Control Release. 2006;111(1-2):19-26.

11. Pistel K, Bittner B, Koll H, Winter G, Kissel T. Biodegradable recombinant human erythropoietin loaded microspheres prepared from linear and star-branched block copolymers: influence of encapsulation technique and polymer composition on particle characteristics. J Control Release. 1999;59(3):309-325.

12. Hahn SK, Oh EJ, Miyamoto H, Shimobouji T. Sustained release formulation of erythropoietin using hyaluronic acid hydrogels crosslinked by Michael addition. Int J Pharm. 2006;322(1-2):44-51.

13. Almeida AJ, Souto E. Solid lipid nanoparticles as a drug delivery system for peptides and proteins. Adv Drug Deliv Rev. 2007;59(6):478-490.

14. Martins BS, Sarmento B, Ferreira DC, Souto EB. Lipid-based colloidal carriers for peptide and protein delivery - liposomes versus lipid nanoparticles. Int J Nanomedicine. 2007;2(4):595-607.

15. Osada K, Christie RJ, Kataoka K. Polymeric micelles from poly(ethylene glycol) -poly(amino acid) block copolymer for drug and gene delivery. $J$ R Soc Interface. 2009;6 Suppl 3:S325-S339.

16. Lee $\mathrm{Y}$, Ishii $\mathrm{T}$, Cabral $\mathrm{H}$, et al. Charge-conversional polyionic complex micelles-efficient nanocarriers for protein delivery into cytoplasm. Angew Chem Int Ed Engl. 2009;48(29):5309-5312.

17. Nishiyama N, Kataoka K. Nanostructured devices based on block copolymer assemblies for drug delivery: Designing structures for enhanced drug function. Polymer Therapeutics II. 2006;193:67-101.

18. Lavasanifar A, Samuel J, Kwon GS. Poly(ethylene oxide)-block-poly(Lamino acid) micelles for drug delivery. Adv Drug Deliv Rev. 2002;54(2): 169-190.

19. Kataoka K, Harada A, Nagasaki Y. Block copolymer micelles for drug delivery: design, characterization and biological significance. Adv Drug Deliv Rev. 2001;47(1):113-131.

20. Layre A, Couvreur P, Chacun H, et al. Novel composite core-shell nanoparticles as busulfan carriers. J Control Release. 2006;111(3):271-280.

21. Discher DE, Ahmed F. Polymersomes. Annu Rev Biomed Eng. 2006;(8): 323-341.

22. Chen GX, Kim HK, Kim ES, Yoon JS. Synthesis of high-molecularweight poly (L-lactic acid) through the direct condensation polymerization of L-lactic acid in bulk state. Eur Polym J. 2006;42(2):468-472.
23. Nagarwal RC, Kant S, Singh PN, Maiti P, Pandit JK. Polymeric nanoparticulate system: a potential approach for ocular drug delivery. $J$ Control Release. 2009;136(1):2-13.

24. Jain AK, Goyal AK, Gupta PN, et al. Synthesis, characterization and evaluation of novel triblock copolymer based nanoparticles for vaccine delivery against hepatitis B. J Control Release. 2009;136(2): 161-169.

25. Garlotta DA. A literature review of poly(lactic acid). J Polym Environ. 2001;9(2):63-84.

26. Chen $\mathrm{C}$, Yu $\mathrm{CH}$, Cheng $\mathrm{YC}$, Yu PH, Cheung MK. Biodegradable nanoparticles of amphiphilic triblock copolymers based on poly (3-hydroxybutyrate) and poly (ethylene glycol) as drug carriers. Biomaterials. 2006;27(27):4804-4814.

27. Li S, Vert M. Synthesis, characterization and stereocomplexInduced gelation of block copolymers prepared by ring-opening polymerization of 1(d)-Lactide in the presence of poly(ethylene glycol). Macromolecules. 2003;36(21):8008-8014.

28. Kataoka K, Harada A, Nagasaki Y. Block copolymer micelles for drug delivery: design, characterization and biological significance. Adv Drug Deliv Rev. 2001;47(1):113-131.

29. Yasugi K, Nagasaki Y, Kato M, Kataoka K. Preparation and characterization of polymer micelles from poly (ethylene glycol)-poly (D, L-lactide) block copolymers as potential drug carrier. J Control Release. 1999;62(1-2):89-100.

30. Porjazoska A, Dimitrov P, Dimitrov I, Cvetkovska M, Tsvetanov CB. Synthesis and aqueous solution properties of functionalized and thermoresponsive poly (D, L-lactide)/polyether block copolymers. Macromol Symp. 2004;210(1):427-436.

31. Yang L, Zhao Z, Wei J, El Ghzaoui A, Li S. Micelles formed by selfassembling of polylactide/poly(ethylene glycol) block copolymers in aqueous solutions. J Colloid Interface Sci. 2007;314(2):470-477.

32. Pierri E, Avgoustakis K. Poly(lactide)-poly(ethylene glycol) micelles as a carrier for griseofulvin. J Biomed Mater Res A. 2005;75(3): 639-647.

33. Kim SY, Shin IG, Lee YM. Preparation and characterization of biodegradable nanospheres composed of methoxy poly(ethylene glycol) and dl-lactide block copolymer as novel drug carriers. J Control Release. 1998;56(1-3):197-208.

34. Domínguez A, Fernandez A, González N, Iglesias E, Montenegro L. Determination of critical micelle concentration of some surfactants by three techniques. J Chem Educ. 1997;74(10):1227-1231.

35. Fang $\mathrm{C}$, Shi B, Pei YY, Hong MH, Wu J, Chen HZ. In vivo tumor targeting of tumor necrosis factor- $\alpha$-loaded stealth nanoparticles: effect of MePEG molecular weight and particle size. Eur J Pharm Sci. 2006; 27(1):27-36.

36. Caliceti P, Salmaso S, Elvassore N, Bertucco A. Effective protein release from PEG/PLA nano-particles produced by compressed gas anti-solvent precipitation techniques. J Control Release. 2004;94(1): 195-205.

37. Harada A, Kataoka K. Novel polyion complex micelles entrapping enzyme molecules in the core: Preparation of narrowly-distributed micelles from lysozyme and poly (ethylene glycol)-poly (aspartic acid) block copolymer in aqueous medium. Macromolecules. 1998;31(2): 288-294.

38. Gao J, Ming J, Gu Z, Zhang X. Investigations on preparation and release behavior in vitro of 9-nitro camptothecin encapsulated micelles. Sheng Wu Yi Xue Gong Cheng Xue Za Zhi. 2008;25(6):1338-1343.

39. Yang KW, Li XR, Yang ZL, Li PZ, Wang F, Liu Y. Novel polyion complex micelles for liver-targeted delivery of diammonium glycyrrhizinate: in vitro and in vivo characterization. $J$ Biomed Mater Res A. 2009;88(1):140-148.

40. Wei S. Biopharmaceutics and Pharmacokinetics. 2nd ed. Beijing: People's Medical Publishing House; 2003:113.

41. Harris JM. Introduction to biotechnical and biomedical applications of poly(ethylene glycol). In: Harris JM, editor. Poly(ethylene glycol) Chemistry: Biochemical and Biomedical Applications. New York: Plenum Press; 1992. 
42. Hinds K, Koh JJ, Joss L, Liu F, Baudys M, Kim SW. Synthesis and characterization of poly(ethylene glycol)-insulin conjugates. Bioconjug Chem. 2000;11(2):195-201.

43. MacDougall IC, Roberts DE, Coles GA, Williams JD. Clinical pharmacokinetics of epoetin (recombinant human erythropoietin). Clin Pharmacokinet. 1991;20(2):99-113.

44. Emmanouel DS, Goldwasser E, Katz AI. Metabolism of pure human erythropoietin in the rat. Am J Physiol. 1984;247(1 Pt 2):F168-F176.

45. Kinoshita H, Ohishi N, Kato M, Tokura S, Okazaki A. Pharmacokinetics and distribution of recombinant erythropoietin in rats. Arzneimittelforschung. 1992;42(2):174-178.
46. Davis ME, Chen ZG, Shin DM. Nanoparticle therapeutics: an emerging treatment modality for cancer. Nat Rev Drug Discov. 2008;7(9): $771-782$.

47. Banerjee A, Onyuksel H. Human pancreatic polypeptide in a phospholipid-based micellar formulation. Pharm Res. 2012;29(6): 1698-1711.

48. Elliott S, Lorenzini T, Chang D, Barzilay J, Delorme E. Mapping of the active site of recombinant human erythropoietin. Blood. 1997;89(2): 493-502.
International Journal of Nanomedicine

\section{Publish your work in this journal}

The International Journal of Nanomedicine is an international, peerreviewed journal focusing on the application of nanotechnology in diagnostics, therapeutics, and drug delivery systems throughout the biomedical field. This journal is indexed on PubMed Central, MedLine, CAS, SciSearch $®$, Current Contents ${ } /$ Clinical Medicine,

\section{Dovepress}

Journal Citation Reports/Science Edition, EMBase, Scopus and the Elsevier Bibliographic databases. The manuscript management system is completely online and includes a very quick and fair peer-review system, which is all easy to use. Visit http://www.dovepress.com/ testimonials.php to read real quotes from published authors.

Submit your manuscript here: http://www.dovepress.com/international-journal-of-nanomedicine-journal 